\title{
A Review Paper on Concrete-Filled Aluminum Tubular Columns
}

\author{
Ahmed Sagban Saadoon Kadhim Zuboon Nasser \\ Department of Civil Engineering, University of Basrah, Iraq

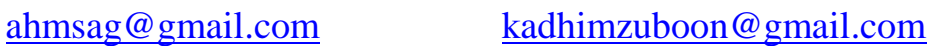

\begin{abstract}
\begin{tabular}{|l|l|l|}
\hline Submission date:- 13/3/2019 & Acceptance date:- 29/5/2019 & Publication date:-12/6/2019 \\
\hline
\end{tabular}
\end{abstract}
\begin{abstract}
The aim of this review paper is to summarize available reports, papers, theses, dissertations and conference papers dealing with the performance of aluminum-concrete composite columns. Hollow aluminum sections filled with concrete have been used as composite columns due to their corrosion resistance, easy production, appearance and lightweight. Many researches were performed in the area of concrete-filled hollow sections (tubes). However, there are few researches have been performed on concrete-filled aluminum tubes. In this review, different available published papers are summarized to view the type of the studied aluminum-concrete columns and the main studied parameters that affecting the behavior of these composite columns. More than (190) specimens are collected and showed in this review.
\end{abstract}

Keywords: Composite columns, Tubular columns, Aluminum hollow sections.

\section{Introduction}

Columns composed of more than one material are usually called composite columns. In these columns, different materials may work together to resist strains and stresses induced by external applied loads. In fact, conventional reinforced concrete columns may be referred as composite columns since they composed of steel and concrete, however the term 'composite columns' is usually used to refer applications such as sections filled with or encased in concrete as shown in Fig. (1). Different materials have been widely used with concrete such as wood, steel, aluminum, FRP and PVC tubes. Hollow aluminum sections filled with concrete have been used as composite columns due to their corrosion resistance, easy production, appearance and lightweight. Due to the low modulus of elasticity of aluminum alloy, the capacity of aluminum columns is not great and it is less than that of steel columns. Filling hollow sections with concrete will increase the capacity of these sections. Thereby the capacity and stiffness of aluminum tubes will be increased by the concrete filling. In general, filling tubes by concrete has many advantages in design and construction, such as [1]:

1. Confining concrete by tubes increases concrete capacity and ductility.

2. Concrete filling prevents local buckling of the tube.

3. Tubes represent a well-distributed reinforcement.

4. Tubes protect concrete from different physical damages.

5. Tubes provide permanent formworks resulting in cost and timesavings.

6. Increasing in columns' load-carrying capacity. 


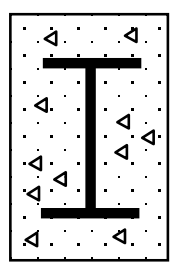

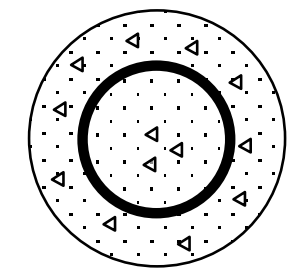

(a) Encased
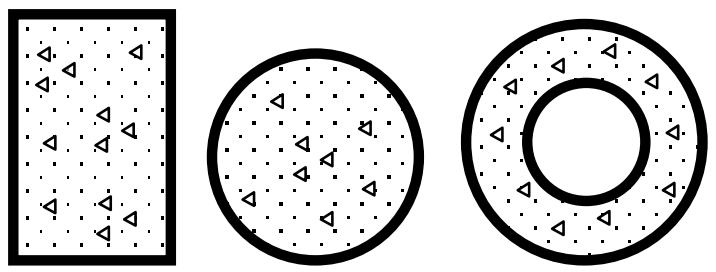

(b) concrete - filled

Figure (1) Different types of composite columns [2]

\section{Literature Review}

Many researches were performed in the area of concrete-filled hollow sections (tubes). Tubes of different shapes and materials were used to confine the concrete core, like steel, FRP and PVC tubes [2 - 4]. However, there are few researches have been performed on concrete-filled aluminum tubes.

Zhou and Young [5], in 2008, investigated experimentally concrete-filled aluminum tubular stub columns under axial compressive loading. Behavior of these columns was studied using different concrete strengths (40, 70 and $100 \mathrm{MPa}$ ) and different tube sections (square and rectangular sections). The used aluminum sections had a nominal proof stress of (240 MPa). The aluminum tube's shape, concrete strength and plate thickness were the main parameters in that study. The range of overall depth/thickness was $(8.2$ - 63.8). Test results were compared with results obtained from the Australian/New Zealand and American standards. It was shown that the design estimations do not agree well with the tests results.

Also Zhou and Young [6], in 2009, studied experimentally concrete-filled circular aluminum tube stub columns under uniform axial compressive loading. Behavior of these columns was studied using different concrete strengths (40, 70 and $100 \mathrm{MPa}$ ) and different tube dimensions. The used aluminum sections had a nominal proof stress of $(240 \mathrm{MPa})$. The aluminum tube's dimensions and concrete strength were the main parameters of that study. The range of diameter/thickness ratio was $(9.7-59.7)$. Test results were compared with results obtained from the Australian/New Zealand and American standards. It was concluded that the results of these standards were generally conservative for these composite columns.

In 2012, Zhou and Young [7] presented a numerical study to analyze and design composite columns, made of concrete-filled circular aluminum tube, using nonlinear finite elements (FE). They developed a FE model and verified it with experimental data. Also, they proposed design equations for these columns based on the composite action between the concrete and aluminum tube. Different crosssection dimensions and material properties were used and (192) numerical data was obtained. Different concrete strengths (40, 70 and $100 \mathrm{MPa}$ ), tube materials (normal strength and high strength material), and $\mathrm{D} / \mathrm{t}$ ratios (ranged from 10 to 160 ) were used. The obtained results were compared with results obtained from the Australian/New Zealand and American standards. It was shown that the suggested design equations can accurately predict the strengths of the concrete-filled circular aluminum tube columns.

Nasser [8], in 2012, carried out an experimental and theoretical investigation on the behavior of concrete-filled circular aluminum tube columns subjected to an axial loading. The used aluminum sections had a nominal proof stress of $(240 \mathrm{MPa})$. The $\mathrm{D} / \mathrm{t}$ and $\mathrm{L} / \mathrm{D}$ ratios were the main studied parameters. Different $\mathrm{D} / \mathrm{t}$ ratios $(11.9 \leq \mathrm{D} / \mathrm{t} \leq 22.8)$ and different $\mathrm{L} / \mathrm{D}$ ratios $(3 \leq \mathrm{L} / \mathrm{D} \leq 10)$ were used and (24) specimens were casted and tested. Empirical equations, to predict the columns' strength were also proposed in that study. It was found that the ratio of experimental to predicted strength has an average value of (1.0104).

In 2014, Resan [9] investigated, experimentally and theoretically, the structural performance of light weight concrete filled circular aluminum tubes under axial compression. The used aluminum sections had a nominal proof stress of (170 MPa). Different loading styles, light weight concrete fashions and concrete strengths were used. It was stated that the results obtained by using the Eurocode and American specifications are in good agreement with the experimental results.

Nasser [10], in 2014, presented an experimental and computational study on the structural performance of concrete filled circular aluminum tube columns under increasing axial loading. The used 
aluminum sections had a nominal proof stress of $(240 \mathrm{MPa})$. Different $\mathrm{D} / \mathrm{t}$ ratios $(23.3 \leq \mathrm{D} / \mathrm{t} \leq 47.8)$ and different $\mathrm{L} / \mathrm{D}$ ratios $(3 \leq \mathrm{L} / \mathrm{D} \leq 10)$ were used and $(24)$ specimens were casted and tested using constant concrete strength of $24.2 \mathrm{MPa}$. Fuzzy inference system (FIS) was also used to predict the ultimate strength of the columns. It was found that the ratio of experimental to predicted strength has an average value of (1.001).

In 2014, Nayak et al. [11] conducted an experimental investigation on self-compacting concrete filled aluminum hollow section tubes under axial compression loading. Different D/t ratio (12-33.3), different L/D ratio (3-10), M25 grade concrete and tubes of $214 \mathrm{MPa}$ yield strength were used. Results have shown that the studied composite columns can withstand a considerable amount of loading.

Resan [12], in 2018, presented an experimental study of light weight concrete filled circular aluminum tube columns enhanced with FRP sheets. Carbon FRP sheets were used to piling the aluminum tubes. Results indicated that the confinement and composite action of different used materials enhanced the ultimate strength, energy dissipation capacity and ductility tested columns. A simplified design equation was also proposed to estimate the ultimate capacity of the studied composite column.

Idan [13], in 2017, used ANSYS software to modeling circular aluminum-concrete composite columns. Previous experimental results were used in verification of the developed ANSYS model. The developed model was then used to study the use of high strength concrete in these composite columns. The findings indicated that, specimens with higher concrete strength (120 MPa) experienced increasing in the ultimate strength and decreasing in ductility.

Al-Mazini and Chkhewier [14], in 2017, studied the performance of composite columns of square and rectangular aluminum tubes filled with concrete. Different D/t ratios (25-62.5), slenderness ratios (3$10)$ and concrete strengths $(25,40$, and $60 \mathrm{MPa})$ were used in that study. Twenty five columns were tested under axial loading. The experimental results showed that the ultimate strength of the tested columns increases as the D/t ratio decreases, and that the concrete strength clearly affects the columns strength. A nonlinear three-dimensional ANSYS model was also developed and used to conduct an analytical investigation. This model gave good results as stated in that study.

In 2018, Zhao et al. [15] used ABAQUS software to present a numerical investigation of circular aluminum-composite (CFAT) stub columns subjected to axial compression. The composite action and nonlinearities of concrete and aluminum materials were considered. Numerical models were developed and validated against available experimental data. Load-deformation relationship, for both concrete and aluminum tube, were also presented. A parametric study, based on the developed FE models, was also presented. The influence of $\mathrm{D} / \mathrm{t}$ ratio, concrete strength and aluminum grades on the ultimate strength of the studied composite columns were investigated in that paper.

Ramanagopal [16], in 2018, conducted an experimental study on the behavior of concrete filled double tube stub columns. Inner steel tubes, different outer tubes (aluminum, stainless steel and mild steel), different concrete strengths $(30,40$ and $50 \mathrm{MPa})$, constant $\mathrm{D} / \mathrm{t}$ ratio (33.3) and constant $\mathrm{L} / \mathrm{D}$ ratio (3) were considered. It was found that the presence of inner tube increases the column strength and it is more beneficial for columns of outer aluminum or stainless steel tubes with low concrete strength.

In 2018, Nachiar et al. [17] studied experimentally the behavior of circular aluminum-concrete composite columns with FRP wrapping. A total of (21) specimens of $(300 \mathrm{~mm})$ height and $(100 \mathrm{~mm})$ diameter with high performance concrete were casted and tested under axial loads. A portion of the columns was wrapped with glass FRP of different layers (single and double) and varying spacing (25, 46.7 and $90 \mathrm{~mm}$ ). ANSYS software was also used to find, analytically, the stiffness of the tested columns.

Joni et al. [18], in 2018, presented an experimental investigation of concrete-filled rectangular aluminum tubular columns retrofitted using basalt strips. Different methods were adapted for retrofitting works. Totally (10) columns of (44.75 x $101.6 \times 1.35 \mathrm{~mm})$ dimensions and (300 mm) height were casted. Some columns were tested to 60,70 , and $80 \%$ of the failure load of a control column. Then, the tested columns were strengthened by (3) layers of basalt strips of $(40 \mathrm{~mm})$ width and different spacings $(25,46$ and $90 \mathrm{~mm}$ ). Finally, the experimental results of normal and retrofitted samples were compared with each other. Results showed that, the performance of columns with less spacing of retrofitting strips is better than that of columns with larger spacing.

The above available published papers are summarized to view the type of the studied aluminumconcrete columns and the main studied parameters that affecting the behavior of these composite columns. More than (190) specimens are collected and showed in this review. Aluminum-concrete 
specimens wrapped, retrofitted or enhanced with other materials like steel sections, FRP sheets, FRP tubes and basalt strips, are not implied herein. Details of concrete filled square, rectangular and circular aluminum tubes are shown in tables (1), (2) and (3), respectively.

Table (1) Details of composite columns with square aluminum tubes

\begin{tabular}{|c|c|c|c|c|c|c|c|c|c|c|}
\hline Ref. & Specimen & $\begin{array}{c}\mathbf{f}_{0.2} \\
(\mathbf{M P a})\end{array}$ & $\begin{array}{c}\mathbf{f}^{\prime} \mathbf{c} \\
(\mathbf{M P a})\end{array}$ & $\begin{array}{l}\text { Depth } \\
\text { D(mm) }\end{array}$ & $\begin{array}{l}\text { Width } \\
\text { B(mm) }\end{array}$ & $\begin{array}{l}\text { Thick. } \\
\text { t(mm) }\end{array}$ & $D / t$ & $\begin{array}{l}\text { Length } \\
\text { L(mm) }\end{array}$ & L/D & $\begin{array}{l}\mathbf{P}_{\text {exp }} \\
(\mathbf{k N})\end{array}$ \\
\hline \multirow{35}{*}{ [5] } & SHS1C0 & 243 & - & 31.9 & 31.9 & 1.96 & 16.2 & 96 & 3.0 & 59 \\
\hline & SHS1C70 & 243 & C70 & 31.9 & 31.9 & 1.96 & 16.3 & 96 & 3.0 & 74 \\
\hline & SHS1C100 & 243 & $\mathrm{C} 100$ & 32.0 & 32.0 & 1.96 & 16.3 & 96 & 3.0 & 85 \\
\hline & SHS2C70 & 226 & C70 & 39.9 & 39.9 & 4.84 & 8.2 & 119 & 3.0 & 175 \\
\hline & SHS2C100 & 226 & $\mathrm{C} 100$ & 39.9 & 39.9 & 4.84 & 8.2 & 120 & 3.0 & 199 \\
\hline & SHS3C0 & 264 & - & 50.7 & 50.7 & 1.97 & 25.7 & 149 & 2.9 & 86 \\
\hline & SHS3C40 & 264 & $\mathrm{C} 40$ & 50.7 & 50.7 & 1.96 & 25.9 & 149 & 3.0 & 131 \\
\hline & SHS3C70 & 264 & $\mathrm{C} 70$ & 50.7 & 50.7 & 1.96 & 25.9 & 150 & 3.0 & 154 \\
\hline & SHS3C 100 & 264 & $\mathrm{C} 100$ & 50.7 & 50.7 & 1.96 & 25.9 & 150 & 3.0 & 228 \\
\hline & SHS4C0 & 268 & - & 50.6 & 50.6 & 3.07 & 16.5 & 150 & 3.0 & 162 \\
\hline & SHS4C40 & 268 & $\mathrm{C} 40$ & 50.6 & 50.6 & 3.08 & 16.4 & 150 & 3.0 & 182 \\
\hline & SHS4C70 & 268 & $\mathrm{C} 70$ & 50.6 & 50.6 & 3.08 & 16.4 & 150 & 3.0 & 203 \\
\hline & SHS4C100 & 268 & C100 & 50.6 & 50.6 & 3.08 & 16.4 & 150 & 3.0 & 261 \\
\hline & SHS5C0 & 222 & - & 63.8 & 63.8 & 2.99 & 21.3 & 192 & 3.0 & 178 \\
\hline & SHS5C0-R & 222 & - & 63.9 & 63.9 & 2.99 & 21.4 & 191 & 3.0 & 178 \\
\hline & SHS5C40 & 222 & $\mathrm{C} 40$ & 63.8 & 63.8 & 3.01 & 21.2 & 191 & 3.0 & 267 \\
\hline & SHS5C70 & 222 & $\mathrm{C} 70$ & 63.8 & 63.8 & 3.01 & 21.2 & 192 & 3.0 & 282 \\
\hline & SHS5C100 & 222 & $\mathrm{C} 100$ & 63.8 & 63.8 & 3.01 & 21.2 & 191 & 3.0 & 332 \\
\hline & SHS6C0 & 246 & - & 76.0 & 76.0 & 3.06 & 24.8 & 227 & 3.0 & 180 \\
\hline & SHS6C40 & 246 & $\mathrm{C} 40$ & 76.0 & 76.0 & 3.09 & 24.6 & 228 & 3.0 & 275 \\
\hline & SHS6C70 & 246 & $\mathrm{C} 70$ & 75.9 & 75.9 & 3.09 & 24.6 & 228 & 3.0 & 382 \\
\hline & SHS6C70- & 246 & $\mathrm{C} 70$ & 75.8 & 75.8 & 3.09 & 24.5 & 227 & 3.0 & 374 \\
\hline & SHS6C100 & 246 & C100 & 76.0 & 76.0 & 3.09 & 24.6 & 228 & 3.0 & 514 \\
\hline & SHS7C0 & 246 & - & 88.1 & 88.1 & 1.77 & 49.7 & 269 & 3.1 & 71 \\
\hline & SHS7C40 & 246 & $\mathrm{C} 40$ & 88.1 & 88.1 & 1.76 & 50.1 & 269 & 3.1 & 299 \\
\hline & SHS7C70 & 246 & $\mathrm{C} 70$ & 88.0 & 88.0 & 1.76 & 50.0 & 264 & 3.0 & 467 \\
\hline & SHS7C100 & 246 & $\mathrm{C} 100$ & 88.0 & 88.0 & 1.76 & 50.0 & 264 & 3.0 & 722 \\
\hline & SHS8C0 & 234 & - & 101.8 & 101.8 & 2.32 & 43.8 & 300 & 2.9 & 137 \\
\hline & SHS8C40 & 234 & $\mathrm{C} 40$ & 101.7 & 101.7 & 2.32 & 43.9 & 300 & 2.9 & 415 \\
\hline & SHS8C70 & 234 & $\mathrm{C} 70$ & 101.6 & 101.6 & 2.32 & 43.8 & 300 & 3.0 & 651 \\
\hline & SHS8C100 & 234 & $\mathrm{C} 100$ & 101.5 & 101.5 & 2.32 & 43.8 & 300 & 2.9 & 1009 \\
\hline & SHS9C0 & 244 & - & 153.1 & 153.1 & 3.32 & 46.2 & 456 & 3.0 & 295 \\
\hline & SHS9C40 & 244 & $\mathrm{C} 40$ & 153.0 & 153.0 & 3.31 & 46.2 & 456 & 3.0 & 915 \\
\hline & SHS9C70 & 244 & $\mathrm{C} 70$ & 152.3 & 152.3 & 3.31 & 46.0 & 456 & 3.0 & 1566 \\
\hline & SHS9C100 & 244 & $\mathrm{C} 100$ & 152.5 & 152.5 & 3.31 & 46.1 & 455 & 3.0 & 2124 \\
\hline \multirow{14}{*}{ [14] } & SHS1 & 240 & M25 & 100.0 & 100.0 & 1.6 & 62.5 & 300.0 & 3.0 & 153 \\
\hline & SHS2 & 240 & M25 & 100.0 & 100.0 & 2 & 50.0 & 300.0 & 3.0 & 176 \\
\hline & SHS3 & 240 & M25 & 100.0 & 100.0 & 3 & 33.3 & 300.0 & 3.0 & 343 \\
\hline & SHS4 & 240 & M25 & 100.0 & 100.0 & 4 & 25 & 300.0 & 3.0 & 480 \\
\hline & SHS5 & 240 & - & 100.0 & 100.0 & 1.6 & 50 & 300.0 & 3.0 & 121 \\
\hline & SHS6 & 240 & M40 & 100.0 & 100.0 & 1.6 & 50 & 300.0 & 3.0 & 214 \\
\hline & SHS7 & 240 & M60 & 100.0 & 100.0 & 1.6 & 50 & 300.0 & 3.0 & 321 \\
\hline & SHS8 & 240 & - & 100.0 & 100.0 & 2 & 50 & 300.0 & 3.0 & 139 \\
\hline & SHS9 & 240 & - & 100.0 & 100.0 & 2 & 50 & 500.0 & 5.0 & 107 \\
\hline & SHS10 & 240 & M25 & 100.0 & 100.0 & 2 & 50 & 500.0 & 5.0 & 140 \\
\hline & SHS11 & 240 & - & 100.0 & 100.0 & 2 & 50 & 700.0 & 7.0 & 77 \\
\hline & SHS12 & 240 & M25 & 100.0 & 100.0 & 2 & 50 & 700.0 & 7.0 & 92 \\
\hline & SHS13 & 240 & - & 100.0 & 100.0 & 2 & 50 & 1000.0 & 10.0 & 69 \\
\hline & SHS14 & 240 & M25 & 100.0 & 100.0 & 2 & 50 & 1000.0 & 10.0 & 81 \\
\hline
\end{tabular}

$\mathrm{f}_{0.2}, \mathrm{D}$, and $\mathrm{t}$ represent static $0.2 \%$ proof stress obtained from tested coupons, outer diameter and thickness of aluminum tube, respectively, L represents length (height) of composite column, $\mathrm{f}_{\mathrm{c}}^{\prime}$ represents compressive strength of concrete ( $\mathrm{C}$ cylinder strength, $\mathrm{M}$ cube strength) and $\mathrm{P}_{\exp }$ represents ultimate compressive strength of composite column. 
Table (2) Details of composite columns with rectangular aluminum tubes

\begin{tabular}{|c|c|c|c|c|c|c|c|c|c|c|}
\hline Ref & Specimen & $\begin{array}{c}\mathbf{f}_{0.2} \\
(\mathbf{M P a}\end{array}$ & $\begin{array}{c}\mathbf{f}^{\prime} \mathbf{c} \\
(\mathbf{M P a}\end{array}$ & $\begin{array}{l}\text { Depth } \\
\text { D(mm }\end{array}$ & $\begin{array}{l}\text { Width } \\
\text { B(mm }\end{array}$ & $\begin{array}{c}\text { Thicknes } \\
\text { s }\end{array}$ & $D / t$ & $\begin{array}{c}\text { Lengt } \\
\mathbf{h}\end{array}$ & L/B & $\begin{array}{c}\mathbf{P} \\
(\mathbf{k N}\end{array}$ \\
\hline \multirow{8}{*}{ [5] } & RHS1C0 & 263 & - & 100.0 & 44.3 & 1.57 & 63. & 300 & 6.7 & 63 \\
\hline & RHS1C40 & 263 & $\mathrm{C} 40$ & 100.0 & 44.1 & 1.57 & 63. & 300 & 6.8 & 182 \\
\hline & RHS1C70 & 263 & $\mathrm{C} 70$ & 100.0 & 44.1 & 1.57 & 63. & 300 & 6.8 & 296 \\
\hline & RHS $1 C 10$ & 263 & $\mathrm{C} 100$ & 99.9 & 44.0 & 1.57 & 63. & 300 & 6.8 & 342 \\
\hline & RHS2C0 & 280 & - & 99.5 & 44.5 & 2.94 & 33. & 300 & 6.7 & 206 \\
\hline & RHS2C40 & 280 & $\mathrm{C} 40$ & 99.6 & 44.7 & 2.90 & 34. & 300 & 6.7 & 237 \\
\hline & RHS2C70 & 280 & $\mathrm{C} 70$ & 95.7 & 44.1 & 2.90 & 34. & 300 & 6.8 & 271 \\
\hline & RHS2C10 & 280 & $\mathrm{C} 100$ & 98.8 & 44.2 & 2.90 & 34. & 300 & 6.7 & 344 \\
\hline \multirow{11}{*}{ [14] } & RHS1 & 240 & M25 & 100.0 & 50.0 & 1.6 & 62. & 300.0 & 6.0 & 70 \\
\hline & RHS2 & 240 & M25 & 100.0 & 50.0 & 2 & 50. & 300.0 & 6.0 & 79 \\
\hline & RHS3 & 240 & M25 & 100.0 & 50.0 & 3 & 33. & 300.0 & 6.0 & 150 \\
\hline & RHS4 & 240 & M25 & 100.0 & 50.0 & 4 & 25 & 300.0 & 6.0 & 200 \\
\hline & RHS5 & 240 & - & 100.0 & 50.0 & 2 & 50 & 300.0 & 6.0 & 64 \\
\hline & RHS6 & 240 & - & 100.0 & 50.0 & 2 & 50 & 500.0 & 6.0 & 562 \\
\hline & RHS70 & 240 & M25 & 100.0 & 50.0 & 2 & 50 & 500.0 & 6.0 & 66 \\
\hline & RHS8 & 240 & - & 100.0 & 50.0 & 2 & 50 & 700.0 & 6.0 & 49 \\
\hline & RHS9 & 240 & M25 & 100.0 & 50.0 & 2 & 50 & 700.0 & 6.0 & 54 \\
\hline & RHS10 & 240 & - & 100.0 & 50.0 & 2 & 50 & 1000.0 & 6.0 & 41 \\
\hline & RHS11 & 240 & M25 & 100.0 & 50.0 & 2 & 50 & 1000.0 & 6.0 & 45 \\
\hline$\lceil 18\rceil$ & ATC & N/A & M25 & 101.65 & 44.75 & 1.35 & 75. & 300.0 & 6.7 & 215 \\
\hline
\end{tabular}

Table (3) Details of composite columns with circular aluminum tubes

\begin{tabular}{|c|c|c|c|c|c|c|c|c|c|}
\hline $\begin{array}{c}\text { Ref } \\
\cdot\end{array}$ & $\begin{array}{c}\text { Speci } \\
\text { men }\end{array}$ & $\begin{array}{c}\text { fo.2 } \\
\text { (MPa) }\end{array}$ & $\begin{array}{c}\mathbf{f}^{\prime} \mathbf{c} \\
(\mathbf{M P a})\end{array}$ & $\begin{array}{l}\text { Diam. } \\
\text { D(mm) }\end{array}$ & $\begin{array}{l}\text { Thick. } \\
\text { t(mm) }\end{array}$ & $D / t$ & $\begin{array}{l}\text { Length } \\
\text { L(mm) }\end{array}$ & L/D & $\begin{array}{l}P_{\exp } \\
(\mathbf{k N})\end{array}$ \\
\hline \multirow{37}{*}{ [6] } & CHS1 & 242.4 & - & 38.0 & 3.91 & 9.7 & 114 & 3.0 & 114.5 \\
\hline & CHS1 & 242.4 & C40 & 38.0 & 3.89 & 9.8 & 114 & 3.0 & 158.9 \\
\hline & CHS1 & 2424 & C70 & 38.0 & 3.90 & 9.7 & 114 & 3.0 & 167.2 \\
\hline & CHS1 & 242.4 & C100 & 38.0 & 3.92 & 9.7 & 114 & 3.0 & 171.5 \\
\hline & CHS2 & 238.4 & - & 50.0 & 3.13 & 16.0 & 150 & 3.0 & 141.2 \\
\hline & CHS2 & 238.4 & $\mathrm{C} 40$ & 50.0 & 3.13 & 16.0 & 150 & 3.0 & 217.0 \\
\hline & CHS2 & 238.4 & C70 & 50.0 & 3.12 & 16.0 & 150 & 3.0 & 238.9 \\
\hline & $\mathrm{CHS} 2$ & 238.4 & C100 & 50.0 & 3.13 & 16.0 & 150 & 3.0 & 327.5 \\
\hline & CSHS3 & 237.8 & - & 60.0 & 2.52 & 23.8 & 180 & 3.0 & 121.3 \\
\hline & CHS3 & 237.8 & C40 & 60.0 & 2.55 & 23.5 & 180 & 3.0 & 244.1 \\
\hline & CHS3 & 237.8 & C70 & 60.0 & 2.54 & 23.6 & 180 & 3.0 & 292.4 \\
\hline & CHS3 & 237.8 & C100 & 59.9 & 2.53 & 23.7 & 180 & 3.0 & 412.6 \\
\hline & CHS4 & 237.0 & - & 76.1 & 2.05 & 37.1 & 228 & 3.0 & 113.4 \\
\hline & CHS4 & 237.0 & C40 & 76.1 & 2.06 & 36.9 & 228 & 3.0 & 329.9 \\
\hline & CHS4 & 237.0 & $\mathrm{C} 70$ & 76.0 & 2.06 & 36.9 & 228 & 3.0 & 415.7 \\
\hline & CHS4 & 237.0 & $\mathrm{C} 100$ & 76.0 & 2.05 & 37.1 & 228 & 3.0 & 611.4 \\
\hline & CHS5 & 244.3 & - & 99.9 & 2.02 & 49.5 & 300 & 3.0 & 162.7 \\
\hline & CHS5 & 244.3 & - & 99.8 & 2.00 & 49.9 & 299 & 3.0 & 160.4 \\
\hline & CHS5 & 244.3 & $\mathrm{C} 40$ & 99.7 & 2.02 & 49.4 & 300 & 3.0 & 543.6 \\
\hline & CHS5 & 244.3 & C70 & 99.8 & 2.06 & 48.4 & 300 & 3.0 & 712.0 \\
\hline & CHS5 & 244.1 & $\mathrm{C} 100$ & 100.0 & 2.05 & 48.8 & 300 & 3.0 & 995.8 \\
\hline & CHS6 & 253.1 & - & 119.7 & 2.55 & 46.9 & 360 & 3.0 & 264.5 \\
\hline & CHS6 & 253.1 & C40 & 119.8 & 2.49 & 48.1 & 360 & 3.0 & 822.8 \\
\hline & CHS6 & 253.1 & C70 & 120.0 & 2.55 & 47.1 & 360 & 3.0 & 1010.3 \\
\hline & CHS6 & 253.1 & C70 & 119.6 & 2.48 & 48.2 & 360 & 3.0 & 1004.0 \\
\hline & CHS6 & 253.1 & $\mathrm{C} 100$ & 119.9 & 2.48 & 48.3 & 360 & 3.0 & 1388.7 \\
\hline & CHS7 & 267.9 & - & 149.8 & 2.51 & 59.7 & 449 & 3.0 & 283.9 \\
\hline & CHS7 & 267.9 & $\mathrm{C} 40$ & 150.1 & 2.53 & 59.3 & 450 & 3.0 & 1111.1 \\
\hline & CHS7 & 267.9 & C70 & 150.1 & 2.54 & 59.1 & 451 & 3.0 & 1496.4 \\
\hline & CHS7 & 267.9 & $\mathrm{C} 100$ & 149.9 & 2.53 & 59.2 & 450 & 3.0 & 2057.8 \\
\hline & CHS8 & 216.9 & - & 150.2 & 4.99 & 30.1 & 448 & 3.0 & 525.8 \\
\hline & CHS8 & 216.9 & C40 & 150.2 & 5.03 & 29.9 & 450 & 3.0 & 1481.9 \\
\hline & CHS8 & 216.9 & C70 & 150.2 & 5.04 & 29.8 & 450 & 3.0 & 1740.6 \\
\hline & CHS8 & 216.9 & C100 & 150.2 & 5.03 & 29.9 & 450 & 3.0 & 2666.1 \\
\hline & $\mathrm{CHS} 9$ & 254.2 & - & 160.2 & 4.01 & 40.0 & 480 & 3.0 & 456.1 \\
\hline & CHS9 & 254.2 & $\mathrm{C} 40$ & 160.1 & 4.03 & 39.7 & 480 & 3.0 & 1494.1 \\
\hline & CHS9 & 254.2 & C70 & 160.5 & 4.07 & 39.4 & 480 & 3.0 & 1974.4 \\
\hline
\end{tabular}




\begin{tabular}{|c|c|c|c|c|c|c|c|c|c|}
\hline & CHS9 & 254.2 & C100 & 160.5 & 4.06 & 39.5 & 480 & 3.0 & 2797.3 \\
\hline & CHS10 & 264.9 & - & 180.2 & 3.75 & 48.1 & 540 & 3.0 & 482.8 \\
\hline & CHS 10 & 264.9 & $\mathrm{C} 40$ & 180.0 & 3.71 & 48.5 & 540 & 3.0 & 1690.2 \\
\hline & CHS10 & 264.9 & $\mathrm{C} 70$ & 180.4 & 3.69 & 48.9 & 540 & 3.0 & 2274.2 \\
\hline & CHS10 & 264.9 & $\mathrm{C} 100$ & 180.5 & 3.75 & 48.1 & 540 & 3.0 & 3139.2 \\
\hline \multirow{24}{*}{ [8] } & D1S3E & 241.4 & - & 38.0 & 3.2 & 11.9 & 114.0 & 3.0 & 104.5 \\
\hline & D1S3 & 241.4 & C24.1 & 38.0 & 3.2 & 11.9 & 114.0 & 3.0 & 148.5 \\
\hline & D1S4 & 241.4 & $\mathrm{C} 24.1$ & 38.0 & 3.2 & 11.9 & 152.0 & 4.0 & 145.8 \\
\hline & D1S6 & 241.4 & C24.1 & 38.0 & 3.2 & 11.9 & 228.0 & 6.0 & 143.7 \\
\hline & D1S8 & 241.4 & C24.1 & 38.0 & 3.2 & 11.9 & 304.0 & 8.0 & 141.9 \\
\hline & D1S10 & 241.4 & C24.1 & 38.0 & 3.2 & 11.9 & 380.0 & 10.0 & 138.9 \\
\hline & D2S3E & 253.6 & - & 50.0 & 3.0 & 16.7 & 150.0 & 3.0 & 121.3 \\
\hline & $\mathrm{D} 2 \mathrm{~S} 3$ & 253.6 & C24.1 & 50.0 & 3.0 & 16.7 & 150.0 & 3.0 & 170.4 \\
\hline & D2S4 & 253.6 & C24.1 & 50.0 & 3.0 & 16.7 & 200.0 & 4.0 & 168.6 \\
\hline & D2S6 & 253.6 & C24.1 & 50.0 & 3.0 & 16.7 & 300.0 & 6.0 & 165.1 \\
\hline & D2S8 & 253.6 & C24.1 & 50.0 & 3.0 & 16.7 & 400.0 & 8.0 & 162.8 \\
\hline & D2S10 & 253.6 & C24.1 & 50.0 & 3.0 & 16.7 & 500.0 & 10.0 & 161.8 \\
\hline & D3S3E & 254.8 & - & 60.0 & 4.2 & 14.3 & 180.0 & 3.0 & 210.1 \\
\hline & D3S3 & 254.8 & C24.1 & 60.0 & 4.2 & 14.3 & 180.0 & 3.0 & 302.7 \\
\hline & D3S4 & 254.8 & C24.1 & 60.0 & 4.2 & 14.3 & 240.0 & 4.0 & 298.5 \\
\hline & D3S6 & 254.8 & $\mathrm{C} 24.1$ & 60.0 & 4.2 & 14.3 & 360.0 & 6.0 & 289.6 \\
\hline & D3S8 & 254.8 & C24.1 & 60.0 & 4.2 & 14.3 & 480.0 & 8.0 & 278.5 \\
\hline & D3S10 & 254.8 & C24.1 & 60.0 & 4.2 & 14.3 & 600.0 & 10.0 & 275.4 \\
\hline & D4S3E & 242.1 & - & 100.0 & 4.4 & 22.8 & 300.0 & 3.0 & 326.4 \\
\hline & D4S3 & 242.1 & C24.1 & 100.0 & 4.4 & 22.8 & 300.3 & 3.0 & 571.4 \\
\hline & D4S4 & 242.1 & C24.1 & 100.0 & 4.4 & 22.8 & 400.4 & 4.0 & 566.7 \\
\hline & D4S6 & 242.1 & $\mathrm{C} 24.1$ & 100.0 & 4.4 & 22.8 & 600.6 & 6.0 & 562.7 \\
\hline & D4S8 & 242.1 & C24.1 & 100.0 & 4.4 & 22.8 & 800.6 & 8.0 & 551.5 \\
\hline & D4S10 & 242.1 & C24.1 & 100.0 & 4.4 & 22.8 & 1001.0 & 10.0 & 545.8 \\
\hline \multirow{17}{*}{ [9] } & A & 170.0 & - & 80.0 & 2.0 & 40.0 & 340 & 4.25 & 103.2 \\
\hline & C1(16. & - & C16.2 L.W & 80.0 & - & - & 340 & 4.25 & 62.4 \\
\hline & C1(20. & - & C20.1 L.W & 80.0 & - & - & 340 & 4.25 & 77.5 \\
\hline & $\mathrm{C} 1(23$. & - & C23.7 L.W & 80.0 & - & - & 340 & 4.25 & 89.0 \\
\hline & $\mathrm{AC} 1(1$ & 170.0 & C16.2 L.W & 80.0 & 2.0 & 40.0 & 340 & 4.25 & 178.6 \\
\hline & $\mathrm{ACl} \#$ & 170.0 & C16.2 L.W & 80.0 & 2.0 & 40.0 & 340 & 4.25 & 165.1 \\
\hline & $\mathrm{AC} 1(2$ & 170.0 & C20.1 L.W & 80.0 & 2.0 & 40.0 & 340 & 4.25 & 208.0 \\
\hline & $\mathrm{AC} 1(2$ & 170.0 & C23.7 L.W & 80.0 & 2.0 & 40.0 & 340 & 4.25 & 221.0 \\
\hline & $\mathrm{C} 2(11$. & - & C11.8 L.W & 80.0 & - & - & 340 & 4.25 & 45.5 \\
\hline & $\mathrm{AC} 2(1$ & 170.0 & C11.8 L.W & 80.0 & 2.0 & 40.0 & 340 & 4.25 & 155.0 \\
\hline & AC2\# & 170.0 & C11.8 L.W & 80.0 & 2.0 & 40.0 & 340 & 4.25 & 135.0 \\
\hline & C3(18. & - & C18.9 L.W & 80.0 & - & - & 340 & 4.25 & 72.8 \\
\hline & $\mathrm{AC} 3(1$ & 170.0 & C18.9 L.W & 80.0 & 2.0 & 40.0 & 340 & 4.25 & 202.3 \\
\hline & AC3\#( & 170.0 & C18.9 L.W & 80.0 & 2.0 & 40.0 & 340 & 4.25 & 188.3 \\
\hline & C4(24) & - & C24 & 80.0 & - & - & 340 & 4.25 & 90.0 \\
\hline & $\mathrm{AC} 4(2$ & 170.0 & $\mathrm{C} 24$ & 80.0 & 2.0 & 40.0 & 340 & 4.25 & 234.0 \\
\hline & AC4\# & 170.0 & C24 & 80.0 & 2.0 & 40.0 & 340 & 4.25 & 222.0 \\
\hline \multirow{24}{*}{ [10] } & $38 \mathrm{~S} 3 \mathrm{E}$ & 243.1 & - & 38.1 & 1.62 & 23.5 & 114.3 & 3.0 & 49.7 \\
\hline & $38 \mathrm{~S} 3$ & 243.1 & C24.2 & 38.1 & 1.62 & 23.5 & 114.3 & 3.0 & 79.8 \\
\hline & 38 S4 & 243.1 & $\mathrm{C} 24.2$ & 38.1 & 1.62 & 23.5 & 152.4 & 4.0 & 78.2 \\
\hline & 38 S6 & 243.1 & $\mathrm{C} 24.2$ & 38.1 & 1.62 & 23.5 & 228.6 & 6.0 & 76.7 \\
\hline & $38 \mathrm{~S} 8$ & 243.1 & C24.2 & 38.1 & 1.62 & 23.5 & 304.8 & 8.0 & 75.6 \\
\hline & $38 \mathrm{~S} 10$ & 243.1 & C24.2 & 38.1 & 1.62 & 23.5 & 381.0 & 10.0 & 74.3 \\
\hline & $50 \mathrm{~S} 3 \mathrm{E}$ & 251.4 & - & 50.2 & 1.6 & 31.4 & 150.6 & 3.0 & 65.2 \\
\hline & $50 \mathrm{~S} 3$ & 251.4 & C24.2 & 50.2 & 1.6 & 31.4 & 150.6 & 3.0 & 130.8 \\
\hline & $50 \mathrm{~S} 4$ & 251.4 & C24.2 & 50.2 & 1.6 & 31.4 & 200.8 & 4.0 & 127.6 \\
\hline & 50 S6 & 251.4 & C24.2 & 50.2 & 1.6 & 31.4 & 301.2 & 6.0 & 124.5 \\
\hline & $50 \mathrm{~S} 8$ & 251.4 & C24.2 & 50.2 & 1.6 & 31.4 & 401.6 & 8.0 & 121.5 \\
\hline & $50 \mathrm{~S} 10$ & 251.4 & C24.2 & 50.2 & 1.6 & 31.4 & 502.0 & 10.0 & 118.5 \\
\hline & 60S3E & 249.7 & - & 60.0 & 2.58 & 23.3 & 180.0 & 3.0 & 120.8 \\
\hline & $60 \mathrm{~S} 3$ & 249.7 & C24.2 & 60.0 & 2.58 & 23.3 & 180.0 & 3.0 & 202.0 \\
\hline & $60 \mathrm{~S} 4$ & 249.7 & C24.2 & 60.0 & 2.58 & 23.3 & 240.0 & 4.0 & 198.7 \\
\hline & 60S6 & 249.7 & $\mathrm{C} 24.2$ & 60.0 & 2.58 & 23.3 & 360.0 & 6.0 & 194.5 \\
\hline & $60 S 8$ & 249.7 & C24.2 & 60.0 & 2.58 & 23.3 & 480.0 & 8.0 & 191.8 \\
\hline & $60 S 10$ & 249.7 & $\mathrm{C} 24.2$ & 60.0 & 2.58 & 23.3 & 600.0 & 10.0 & 189.6 \\
\hline & $100 \mathrm{~S} 3$ & 241.7 & - & 100.3 & 2.1 & 47.8 & 300.9 & 3.0 & 165.4 \\
\hline & $100 \mathrm{~S} 3$ & 241.7 & $\mathrm{C} 24.2$ & 100.3 & 2.1 & 47.8 & 300.9 & 3.0 & 420.7 \\
\hline & $100 \mathrm{~S} 4$ & 241.7 & $\mathrm{C} 24.2$ & 100.3 & 2.1 & 47.8 & 401.2 & 4.0 & 414.7 \\
\hline & $100 S 6$ & 241.7 & $\mathrm{C} 24.2$ & 100.3 & 2.1 & 47.8 & 601.8 & 6.0 & 406.9 \\
\hline & 100S 8 & 241.7 & C24.2 & 100.3 & 2.1 & 47.8 & 802.4 & 8.0 & 402.3 \\
\hline & $100 \mathrm{~S} 10$ & 241.7 & C24.2 & 100.3 & 2.1 & 47.8 & 1001.0 & 10.0 & 398.5 \\
\hline
\end{tabular}




\begin{tabular}{|c|c|c|c|c|c|c|c|c|c|}
\hline \multirow{8}{*}{ [11] } & $\mathrm{E}(\mathrm{G} 1) 3$ & 214.0 & - & 38.1 & 3.17 & 12.0 & 115.0 & 3.0 & 83.39 \\
\hline & (G1)3 & 214.0 & M28.1 S.C & 38.1 & 3.17 & 12.0 & 115.0 & 3.0 & 122.63 \\
\hline & $\mathrm{E}(\mathrm{G} 2) 3$ & 214.0 & - & 50.8 & 3.17 & 16.0 & 153.0 & 3.0 & 112.8 \\
\hline & $(\mathrm{G} 2) 3$ & 214.0 & M28.1 S.C & 50.8 & 3.17 & 16.0 & 153.0 & 3.0 & 181.49 \\
\hline & $\mathrm{E}(\mathrm{G} 3) 3$ & 214.0 & - & 63.0 & 3.17 & 19.9 & 189.0 & 3.0 & 83.39 \\
\hline & $(\mathrm{G} 3) 3$ & 214.0 & M28.1 S.C & 63.0 & 3.17 & 19.9 & 189.0 & 3.0 & 191.3 \\
\hline & $\mathrm{E}(\mathrm{G} 4) 3$ & 214.0 & - & 100.0 & 3.0 & 33.3 & 300.0 & 3.0 & 181.49 \\
\hline & $(\mathrm{G} 4) 3$ & 214.0 & M28.1 S.C & 100.0 & 3.0 & 33.3 & 300.0 & 3.0 & 539.55 \\
\hline \multirow{3}{*}{ [12] } & A & 170.0 & - & 80.0 & 2.0 & 40.0 & 300.0 & 3.75 & 102.7 \\
\hline & $\mathrm{C}$ & - & C20 S.C & 80.0 & & - & 300.0 & 3.75 & 74.0 \\
\hline & $\mathrm{AC}$ & 170.0 & C20 S.C & 80.0 & 2.0 & 40.0 & 300.0 & 3.75 & 1187.72 \\
\hline \multirow{3}{*}{ [16] } & AL- & 240.0 & M30 & 100.0 & 3.0 & 33.3 & 300.0 & 3.0 & 340 \\
\hline & AL- & 240.0 & M40 & 80.0 & 2.0 & 40.0 & 300.0 & 3.75 & 480 \\
\hline & AL- & 240.0 & M50 & 80.0 & 2.0 & 40.0 & 300.0 & 3.75 & 700 \\
\hline$[18]$ & $\mathrm{SC}$ & N/A & M87.6 H. P & 100.0 & 3.0 & 33.3 & 300.0 & 3.0 & 728.6 \\
\hline
\end{tabular}

\section{Statistical Survey}

In order to know where is a lack of researches in studying some parameters that affecting the behavior of aluminum-concrete composite columns, the numbers of previous studies dealt with different parameters (concrete strength, tube's dimensions, D/t ratio and L/D ratio) are counted and presented in Fig. (2). It is obvious from this figure that there is a lack of experimental investigations on some areas, like the use of high strength concrete, rectangular aluminum tubes of low and high L/W ratio, and large size specimens. Hence, more investigations on the structural performance of these composite columns are needed.

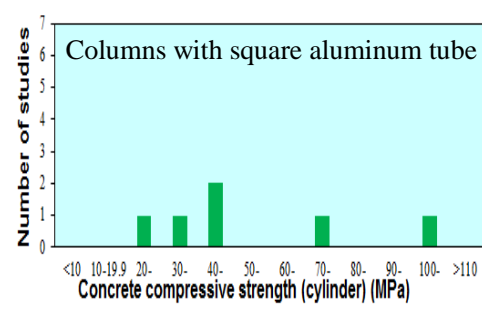

(a)

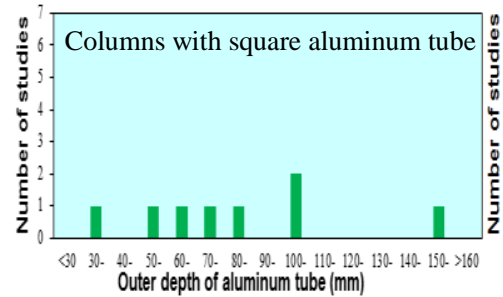

(d)

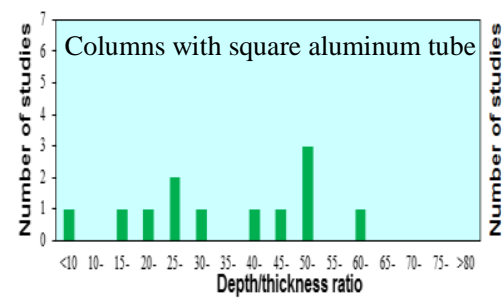

(g)

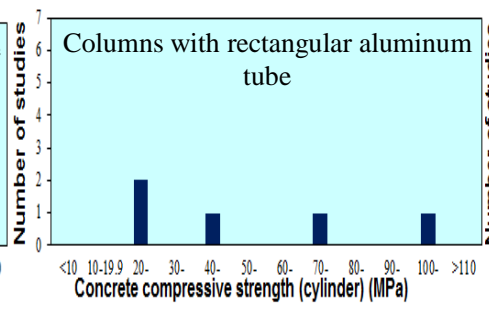

(b)

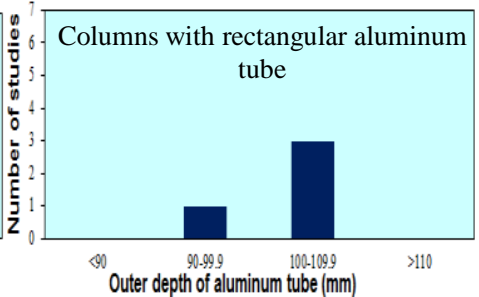

(e)

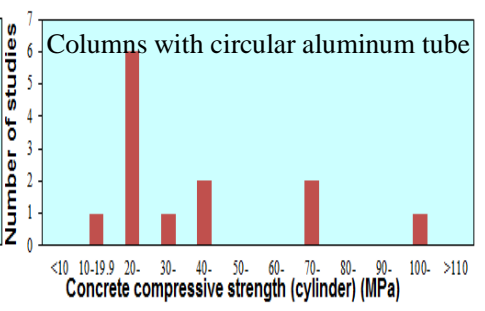

(c)

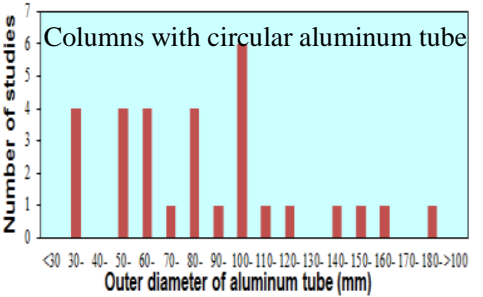

(f)

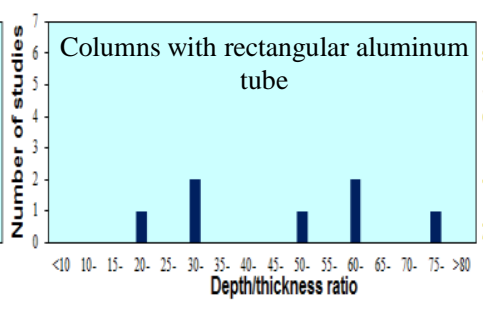

(h)

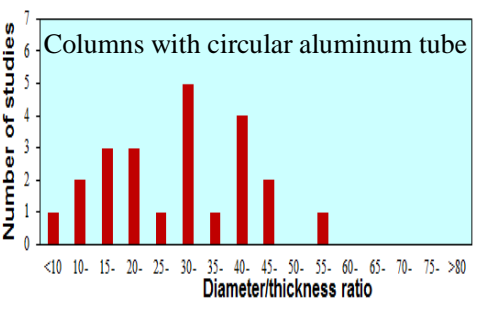

(i) 


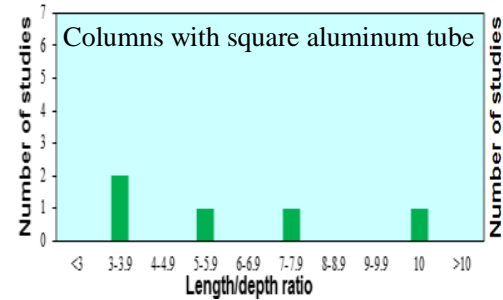

(j)

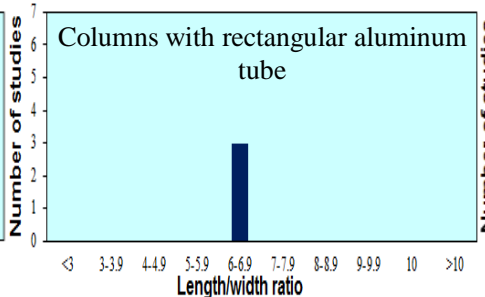

$(\mathrm{k})$

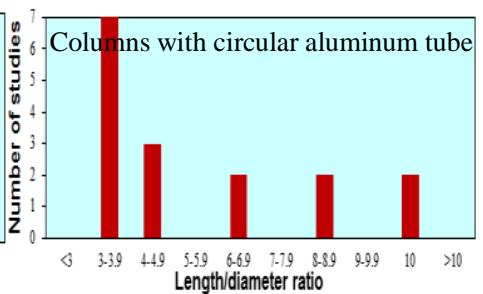

(1)

Figure (2) Number of studies dealt with different parameters

\section{Conclusions and Recommendations}

The reviewed literature demonstrates that the behavior of aluminum-concrete composite columns depends on various parameters, such as loading style, stress-strain relationship of individual materials, $\mathrm{D} / \mathrm{t}$ ratio, $\mathrm{L} / \mathrm{D}$ ratio, .... etc. The aluminum tube provided a considerably sufficient support (confinement) to the concrete core and increased the ultimate strength of aluminum-concrete columns. The aluminumconcrete columns have considerably good corrosion resistance, strength and ductility prior to failure. There is a lack of experimental investigations on some areas, like the use of high strength concrete, rectangular aluminum tubes of low and high $\mathrm{L} / \mathrm{W}$ ratio, and and large size specimens. Hence, more investigations on the structural performance of these composite columns are needed. The following, are some further possibilities for proposed works:

A. Using different types of concrete filling like high strength, self-compacting, light-weight and recycled aggregate concrete.

B. Using composite columns of large size to investigate the size effect on the performance of these columns.

C. Studying the durability of aluminum-concrete columns.

D. Studying the behavior of these composite columns under fire.

\section{CONFLICT OF INTERESTS.}

- There are no conflicts of interest.

\section{References}

[1] Becque, J., "Analytical modeling of concrete columns confined by FRP", M. Sc. Thesis, Department of Civil and Geological Engineering, University of Manitoba, 2000.

[2] Saadoon A. S., "Experimental and theoretical investigation on PVC-concrete composite columns", Ph. D. Dissertation, Department of Civil Engineering, University of Basrah, Iraq, 2010.

[3] Lam, D., and Wong, K.K.Y., "Axial capacity of concrete filled stainless steel columns", ASCE Journal of Structures 2005, pp. 1107-1120.

[4] Hong, W.K., and Kim, H.C., "Behavior of concrete columns confined by carbon composite tubes", Canadian Journal of Civil Eng., 31(2):178-188, 2004.

[5] Zhou F. and Young B., "Tests of concrete-filled aluminum stub columns" Thin-Walled Structures, 46(6):573-583, June 2008.

[6] Zhou F. and Young B., "Concrete-filled aluminum circular hollow section column tests", ThinWalled Structures, 47(11):1272-1280, November 2009.

[7] Zhou F. and Young B., "Numerical analysis and design of concrete-filled aluminum circular hollow section columns", Thin-Walled Structures, 50(1): 45-55, January 2012.

[8] Nasser K. Z., "Structural behavior of concrete filled aluminum tubular columns", Basrah Journal for Engineering Science, 2012, pp 46-59. 
[9] Resan S. F., "Experimental investigation of aluminum-light weight concrete composite columns", Basrah Journal for Engineering Science, 14(1):13-25, 2014.

[10] Nasser K. Z., "Experimental and computational study of concrete fill aluminum tubular column under axial loads", Kufa Journal of Engineering (K.J.E), 5(2):29-46, June 2014.

[11] Nayak G., Shetty K. K. and Abdalla S., "Effect of depth to thickness ratio and length to depth ratio on hollow section aluminium tubes filled with self-compacting concrete", International Journal of Research in Engineering and Technology, 3(3):675-678, May 2014.

[12] Resan S. F., "Behavior of aluminum columns enhanced with CFRP and filled by light weight concrete", Engineering and Technology Journal, 32(11 Part A Eng.): 2658-2672, 2014.

[13] Idan F. K., "Finite element analysis of concrete-filled aluminum tube columns", International Journal of Applied Engineering Research, 12(12):3054-3062, 2017.

[14] Al-Mazini M. A. and Chkhewier A. H., "Behavior of concrete filled aluminum square and rectangular hollow section columns under axial loads: Experimental and analytical study", Journal of Babylon University/Engineering Sciences, 25(2):712-726, 2017.

[15] Zhao H. Y., Wang F. C. and Hana L. H., "Analytical behavior of concrete-filled aluminum tubular stub columns under axial compression", 12th International Conference on Advances in SteelConcrete Composite Structures (ASCCS 2018), València, Spain, June 27-29, pp 373-378, 2018.

[16] Ramanagopal S., "Case study on double tube stub columns", Indian Journal of Science and Technology, 11(21):1-9, June 2018.

[17] Nachiar S., Muthukumar S. and Anandh S., "Effect of retreofitting on high performance geo polymer concrete filled aluminium tubular section using glass fibre wrapping", International Journal of Engineering \& Technology, 7 (2.33):1126-1128, 2018.

[18] Joni M., Rexin C., Mengesha A. and Kassa H. K., "An experimental investigation on concrete filled aluminium tubular section retrofitted by using basalt strips", International Research Journal of Engineering and Technology (IRJET), 5(4):2270-2276, April 2018. 


\section{مراجعة حول أعمدة أنابيب الالمنيوم المملوية بالخرسانة

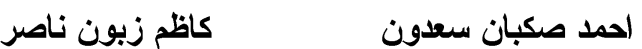 \\ قسم الهنسة الدنبة، كلية الهنسسة، جامعة البصرة، العراق}

kadhimzuboon@gmail.com ahmsag@gmail.com

\section{الخلاصة}

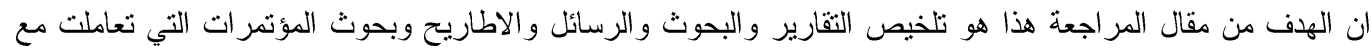

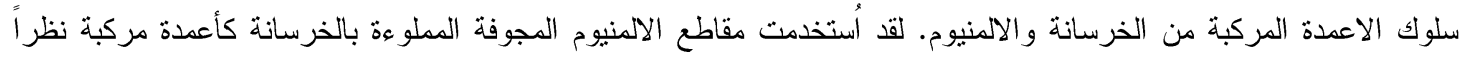

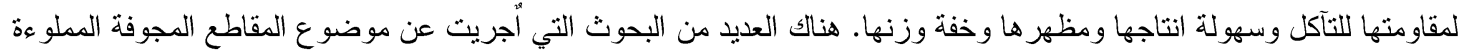

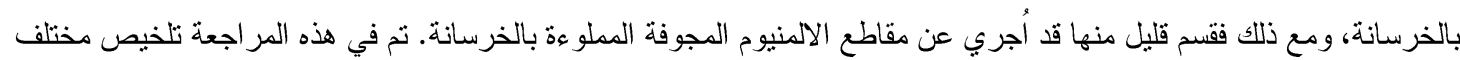
البحوث المنشورة والمتوفرة عن هذا الموضوع وذلك لبيان نوع أعمدة الالمنيوم-الخرسانة المدروسة والمتنغيرات الرئيسة المؤثرة على سلوك هذه الاعمدة المركبة. وقد نم جمع وعرض أكثر من (190) نموذج في هذه المر اجعة. كلمات الاداله: أعمدة مركبة، أعمدة انبويية، مقاطع المنيوم مجوفة. 